01,02

\title{
Параметры туннельного барьера сверхпроводниковых структур на основе ниобия
}

\author{
() М.Е. Парамонов, Л.В. Филиппенко, П.Н. Дмитриев, М.Ю. Фоминский, А.Б. Ермаков, В.П. Кошелец \\ Институт радиотехники и электроники им. В.А. Котельникова РАН, \\ Москва, Россия \\ E-mail: paramonov@hitech.cplire.ru
}

Поступила в Редакцию 26 марта 2020 г.

В окончательной редакции 26 марта 2020 г.

Принята к публикации 2 апреля 2020 г.

\begin{abstract}
Проведена оценка основных параметров туннельного барьера джозефсоновских переходов $\mathrm{Nb} / \mathrm{AlO}_{x} / \mathrm{Nb}$ и $\mathrm{Nb} / \mathrm{AlN} / \mathrm{Nb}$ в широком диапазоне значений плотностей тока с использованием метода Симмонса. Экспериментально определены зависимости высоты и ширины туннельного барьера от удельного сопротивления для каждого типа переходов. Снижение высоты туннельного барьера перехода с прослойкой из AlN на $0.3 \mathrm{eV}$, по сравнению с оксидным, позволяет получать переходы с плотностью тока выше $15 \mathrm{kA} / \mathrm{cm}^{2}$ при технологически достижимой толщине изоляционного слоя порядка $10 \AA$, что дает возможность реализовывать параметр качества $R_{j} / R_{n}$ не ниже 25 .
\end{abstract}

Ключевые слова: сверхпроводимость, сверхпроводниковый туннельный переход, параметры туннельного барьера, метод Симмонса, приемные устройства терагерцового диапазона.

DOI: 10.21883/FTT.2020.09.49755.31H

\section{1. Введение}

Смесители на основе туннельных переходов сверхпроводник-изолятор-сверхпроводник (СИС) являются наиболее чувствительными входными элементами для когерентных приемников на частотах от 0.1 до $1.2 \mathrm{THz}$, их шумовая температура ограничена только квантовым пределом. В настоящее время СИС-приемники используются в качестве штатных устройств на большинстве как наземных, так и космических радиотелескопов во всем мире [1-6]. Для повышения рабочей частоты сверхпроводящих схем, основанных на СИС-переходах, и расширения их полосы, необходимы туннельные переходы с высокой плотностью тока. Однако существует предел повышения прозрачности барьера для СИС-переходов на основе окиси алюминия. Это предел составляет величину порядка $10-15 \mathrm{kA} / \mathrm{cm}^{2}$, при дальнейшем увеличении плотности тока наступает резкая деградация качества переходов [7-10] (см. рис. 1). Для того, чтобы преодолеть это ограничение была разработана технология изготовления туннельных СИС-переходов с барьером из $\mathrm{AlN}$, который формируется нитридизацией поверхности Al в плазменном RF-разряде в среде чистого $\mathrm{N}_{2}$ [4-6,10-12]. Новый тип переходов позволил получить значения плотностей тока более $15 \mathrm{kA} / \mathrm{cm}^{2}$ для реализации широкополосных приемников и генераторов THz-диапазона. Важной особенностью переходов с прослойкой из AIN является возможность ее использования совместно с верхним электродом из $\mathrm{NbN}$, что позволяет увеличить щелевое напряжение перехода с 2.8 до $3.7 \mathrm{mV}$ [10] и повысить верхнюю частотную границу работы СВЧ-устройств.

\section{2. Методы изготовления туннельных структур}

Для изготовления СИС-переходов на основе структур $\mathrm{Nb} / \mathrm{AlO}_{x} / \mathrm{Nb}$ и $\mathrm{Nb} / \mathrm{AlN} / \mathrm{Nb}$ использовалась технология селективного травления и анодизации ниобия - SNEAP (от англ. - Selective Niobium Etching and Anodization

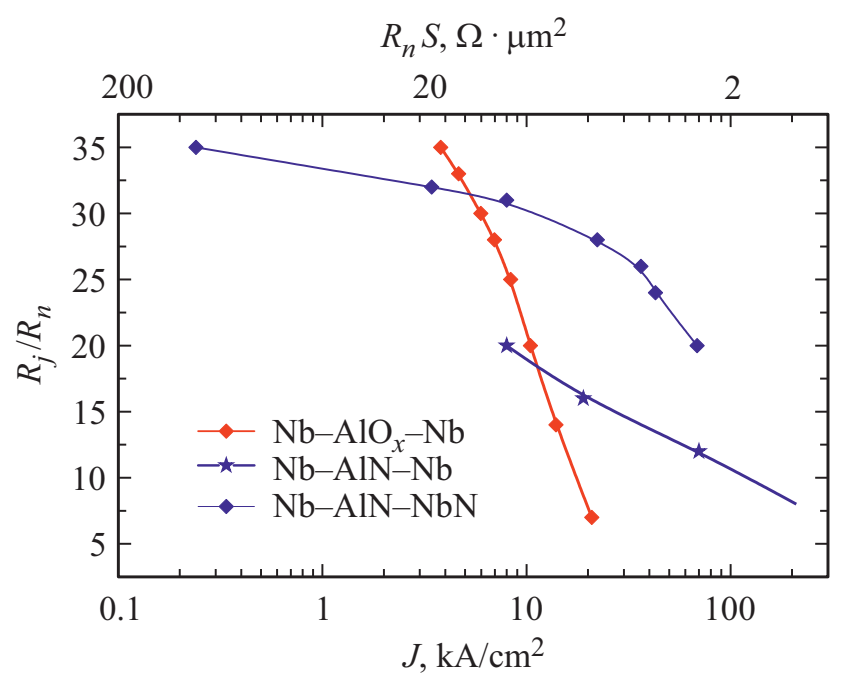

Рис. 1. Зависимости отношения параметра качества $R_{j} / R_{n}$ от значения плотности туннельного тока СИС-перехода $J[10]$. Значение $J$ для данных переходов рассчитано из вида ВАХ (величина щелевого напряжения $V_{g}$ и нормального сопротивления $R_{n}$ ). Более универсальной величиной является значение удельного сопротивления перехода $R_{n} S$, выраженное в единицах $\Omega \cdot \mu \mathrm{m}^{2}$; поэтому в дальнейшем будет использоваться именно величина $R_{n} S$. 
Process) [13,14]. Ниже приведено описание процесса изготовления туннельных СИС-переходов, разработанного в ИРЭ им. В.А. Котельникова РАН и используемого для изготовления приемных структур THz-диапазона $[10,15,16]$. В качестве подложки для изготовления тестовых структур обычно используются пластины из кремния размером $24 \times 24 \mathrm{~mm}$. Для предотвращения травления материала подложки в процессе формирования переходов плазмохимическим травлением проводится магнетронное напыление буферного стоп-слоя $\mathrm{Al}_{2} \mathrm{O}_{3}$ толщиной порядка $100 \mathrm{~nm}$. Далее на подложке формируется фоторезистивная маска, определяющая геометрию базового электрода под взрывную литографию. Следующим этапом является напыление многослойной структуры, определяющей структуру СИС-перехода.

Напыление проводится магнетронным методом в едином вакуумном цикле в сверхвысоковакуумной установке с остаточным давлением $10^{-6} \mathrm{~Pa}$, оборудованной криогенным и турбомолекулярными насосами и оснащенной водоохлаждаемым держателем подложки системами магнетронного распыления на постоянном токе (DC) и на частоте $13.56 \mathrm{MHz}(\mathrm{RF})$. После загрузки подложек в установку и откачки, производится предварительная ионная очистка поверхности образцов от органических загрязнений. Далее осаждается слой нижнего $\mathrm{Nb}$ толщиной $200 \mathrm{~nm}$, затем слой барьерного $\mathrm{Al}$ толщиной $7 \mathrm{~nm}$. Ниобий и алюминий осаждались методом реактивного DC-напыления в среде $\mathrm{Ar}$.

Для получения барьера из окисла алюминия поверхность алюминия окисляется в атмосфере чистого кислорода и осаждается слой верхнего $\mathrm{Nb}$ толщиной 50-100 nm. Для получения туннельной структуры с барьером AlN на нижний электрод из ниобия толщиной $200 \mathrm{~nm}$ методом магнетронного распыления наносится тонкий слой алюминия толщиной 5-7 nm. Как было показано ранее, см., например, [17], тонкая пленка $\mathrm{Al}$ (толщиной более $3 \mathrm{~nm}$ ) равномерно покрывает поверхность ниобия, предотвращая его окисление. Затем часть слоя алюминия нитридизируется в плазме из чистого азота, причем возможно получение требуемой толщины туннельного барьера путем варьирования мощности разряда и времени нитридизации. Малая мощность разряда и большое расстояние от мишени до образца позволило избежать как повреждения туннельного барьера высокоэнергетическими ионами, так и дополнительного напыления материала мишени в процессе нитридизации. Как и в случае термического окисления, оставшийся тонкий слой алюминия является сверхпроводящим из-за эффекта близости с ниобием, поскольку длина когерентности в алюминии много больше толщины этого слоя. Следующей операцией производится напыление верхнего электрода из ниобия или нитрида ниобия $(\mathrm{NbN})$ толщиной $150 \mathrm{~nm}$. Окончательно базовый электрод формируется путем удаления пленки фоторезиста, покрытой напыленной многослойной структурой, с незакрытых базовым электродом участков схемы в ацетоне или диметилформамиде с применением ультразвука (взрывная литография).
Для формирования геометрии СИС-переходов использовалась установка оптической фотолитографии с разрешением $0.7 \mu \mathrm{m}$, что позволяет получать переходы площадью менее $1 \mu \mathrm{m}^{2}$. Переходы формируются плазмохимическим травлением в среде $\mathrm{CF}_{4}$ путем удаления слоя верхнего $\mathrm{Nb}$ многослойной структуры по маске из фоторезиста определяющей геометрию переходов. В качестве стоп-слоя, предотвращающего дальнейшее травление структуры, выступает барьерный слой $\mathrm{AlO}_{x}$ или AlN. После плазмохимического травления производится анодирование по той же маске фоторезиста и напыляется RF-магнетронным методом изоляционный слой $\mathrm{SiO}_{2}$, типичная толщина которого составляет $250 \mathrm{~nm}$. Анодизация необходима для обеспечения более надежной изоляции по периметру СИС-переходов во избежание возможных закороток между базовым и верхним подводящим электродами в этих областях. Вскрытие контактов к переходам осуществляется методом взрывной литографии. Верхний подводящий электрод так же формируется методом взрывной литографии путем напыления слоя $\mathrm{Nb}$ толщиной 300-500 nm по маске фоторезиста с последующим его удалением в растворителях. Аналогичным способом формируются области контактных площадок, в качестве материала которых обычно используется золото. Более подробно процессы изготовления описаны в работах [18-20].

Изготовленные таким образом структуры использовались при создании приемных устройств для радиоастрономии $[5,6,16]$, сверхпроводниковых интегральных приемников для исследования атмосферы [21,22], а также сверхпроводниковых генераторов с передающей щелевой антенной $[23,24]$ для передачи THz-излучения в открытое пространство.

\section{3. Расчет параметров туннельного барьера}

Для создания THz-смесителя с малыми потерями и, как следствие, низким уровнем собственных шумов, емкость СИС-перехода должна быть скомпенсирована введением дополнительной индуктивности. Оценка емкости перехода возможна после определения его основных электрических и геометрических параметров. Основными характеристиками туннельного барьера типа металлизолятор-металл являются средняя высота барьера $\bar{\varphi}$ и его ширина $d$ (см. рис. 2), по этим параметрам можно судить о характеристиках изготовленных переходов. Одним из таких параметров является толщина изоляционного слоя $d$ в структурах $\mathrm{Nb} / \mathrm{AlN} / \mathrm{Nb}(\mathrm{NbN})$, который формируется путем нитридизации алюминия в процессе изготовления СИС-смесителя.

Существует универсальный метод определения толщины туннельного барьера $d$, а также его средней высоты $\bar{\varphi}$ из ВАX при больших напряжениях, основанный на измерении зависимости плотности туннельного тока от напряжения. Эта зависимость была предложена Симмонсом [25] и обобщена Бринкманом [26] для туннельных переходов в пределе малых напряжений смещения. 


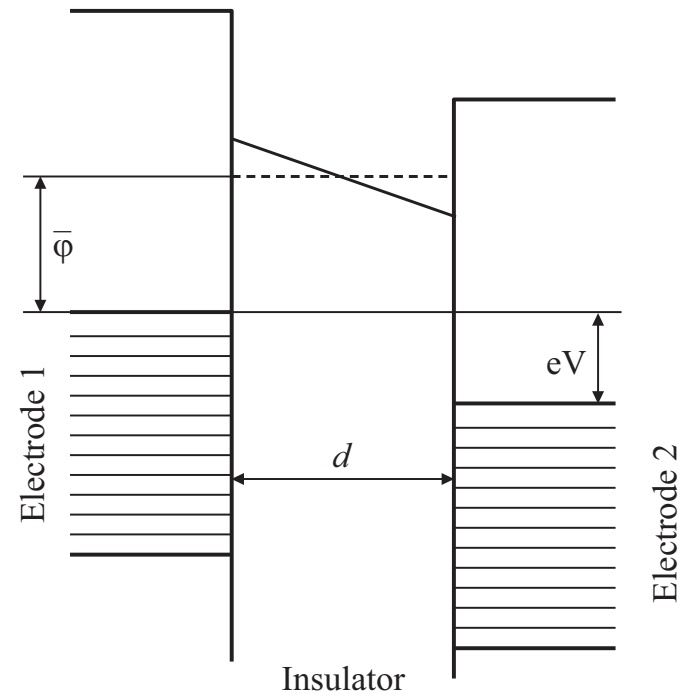

Рис. 2. Схематическое изображение симметричного потенциального барьера в туннельном переходе. $\bar{\varphi}-$ среднее значение высоты барьера, $d$ - ширина барьера, $e V$ - величина приложенного напряжения к электродам.

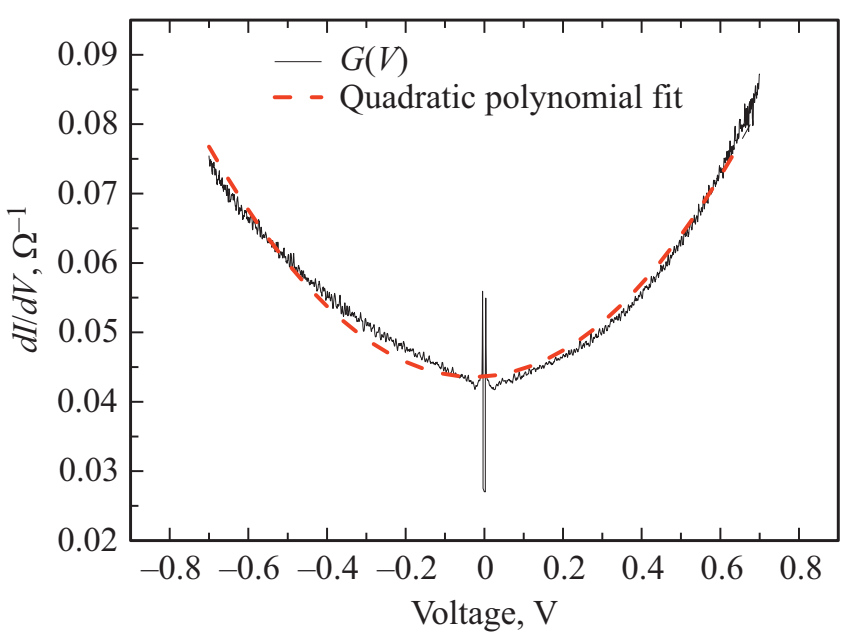

Рис. 3. Зависимость дифференциальной проводимости туннельного СИС-перехода $d I / d V$ от напряжения (сплошная кривая) и аппроксимация квадратичным полиномом (штриховая кривая).

Следует также отметить, что большое значение при расчетах имеет параметр эффективной массы электрона в барьере, равный примерно половине массы электрона в металле [27]. Расчетные формулы и методика подробно изложены в работе [28]. Важно учесть, что в работе [28] уравнение (2):

$$
G=d I / d V=\alpha\left(1+3 \gamma V^{2}\right)
$$

является упрощенной формой выражения

$$
G(V)=\alpha+2 \beta V+3 \gamma V^{2}+4 \delta V^{3}+\ldots,
$$

где $\alpha, \beta, \gamma, \delta-$ величины, в теоретической модели, характеризующие аппроксимационную кривую и косвен- но зависящие от основных параметров барьера. Rowell в своей работе [29] указывает на тот факт, что при обработке экспериментальных данных и аппроксимации, с увеличением диапазона напряжений приложенных к переходу возрастает значимость более высоких степеней $V$ в теории Симмонса. Используя в качестве аппроксимационной функции для переходов $\mathrm{Nb} / \mathrm{AlN} / \mathrm{Nb}$ полином 2-ой степени - теоретическая кривая в целом повторяет характер кривой за исключением некоторых областей (рис. 3). Особенности в области малых напряжений обусловлены наличием сверхпроводниковой щели.

Применяя для аппроксимации полином 3-ей степени, теоретическая кривая демонстрирует практически полное соответствие эксперименту. Проводя количествен-

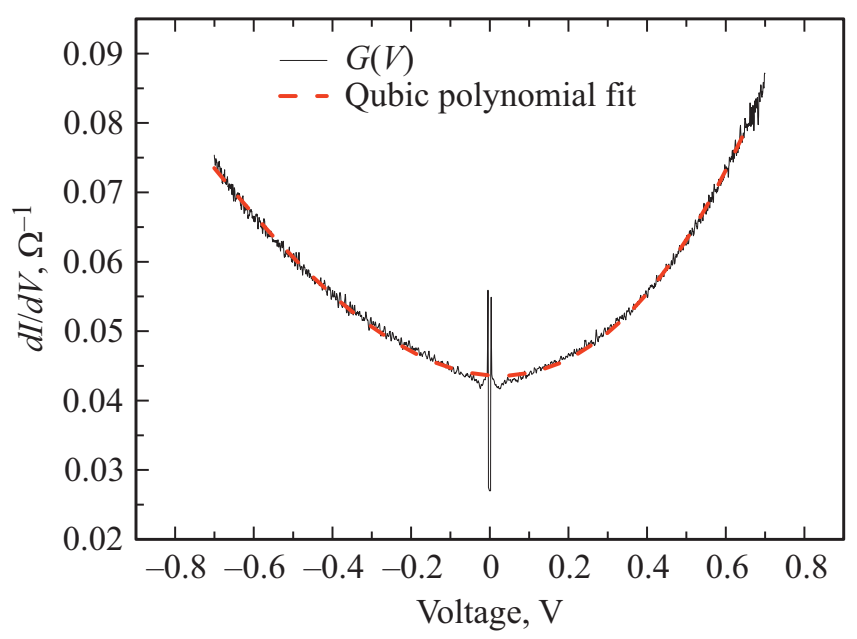

Рис. 4. Зависимость дифференциальной проводимости туннельного СИС-перехода $d I / d V$ от напряжения (сплошная кривая) и аппроксимация кубическим полиномом (штриховая кривая).

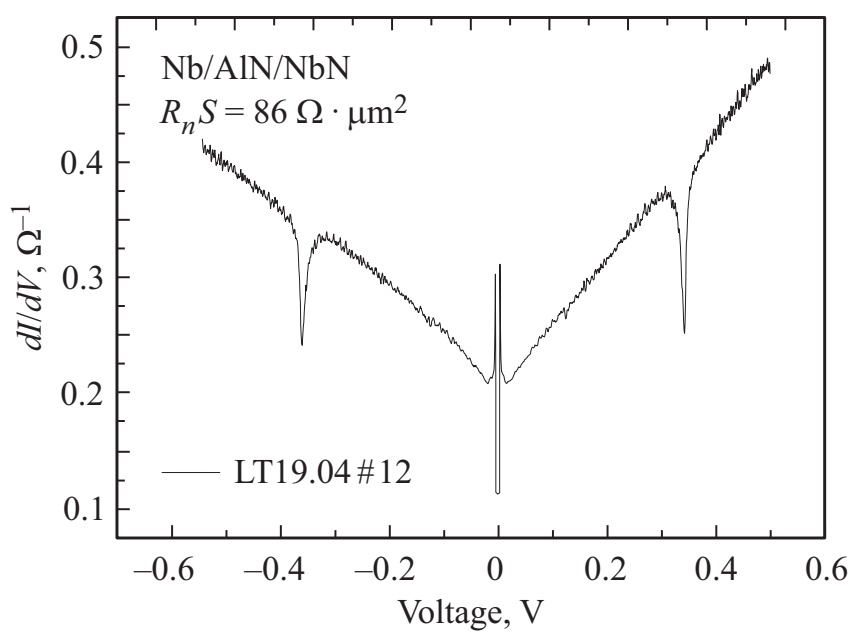

Рис. 5. Дополнительные особенности на характеристиках туннельных переходов в области напряжений сотни милливольт, связанные с эффектом проскальзывания фазы или формированием цепочки движущихся абрикосовских вихрей в пленках сверхпроводящих электродов. 
ную оценку соответствия теории и эксперимента, коэффициент детерминации для кривой полинома 3-ей степени равен 0.99, а для полинома 2-ой степени 0.97, см. рис. 4. Использование в расчетах такой аппроксимации позволило проводить более точную оценку параметров $\bar{\varphi}$ и $d$, минимизировав расчетную ошибку.

У некоторых измеренных образцов с сопротивлением $10 \Omega$ и меньше на зависимости $d I / d V$ от $V$ были видны резкие провалы проводимости на одинаковых напряжениях разной полярности. Для примера, на рис. 5 показана кривая $d I / d V(V)$ для перехода с $R_{n} S=240 \Omega \cdot \mu \mathrm{m}^{2}$ и сопротивлением $3 \Omega$. Подобные особенности сильно искажали параболический характер кривой и порой делали невозможным дальнейшую оценку параметров. Это явление можно объяснить эффектом проскальзыванием фазы, либо формированием цепочки движущихся абрикосовских вихрей, образованных в результате частичного разрушения сверхпроводимости в пленках сверхпроводящих электродов (низкоомные переходы требуют большие токи для достижения необходимого напряжения порядка $0.5 \mathrm{~V})$.

\section{4. Результаты}

По данным полученным в результате расчетов были построены зависимости средней высоты и ширины туннельного барьера от значения удельного сопротивления $R_{n} S$ для двух типов барьеров, см. рис. 6 и 7. Видно, что для больших значений плотности тока (малые $R_{n} S$ ) толщина туннельного слоя для барьера AlN существенно больше по сравнению с оксидным барьером. Данные для переходов $\mathrm{Nb} / \mathrm{AlO}_{x} / \mathrm{Nb}$ взяты из работы [27].

Следует отметить, что использование в расчетах параметров барьера поправки на эффективную массу электрона сильно влияет на конечный результат.

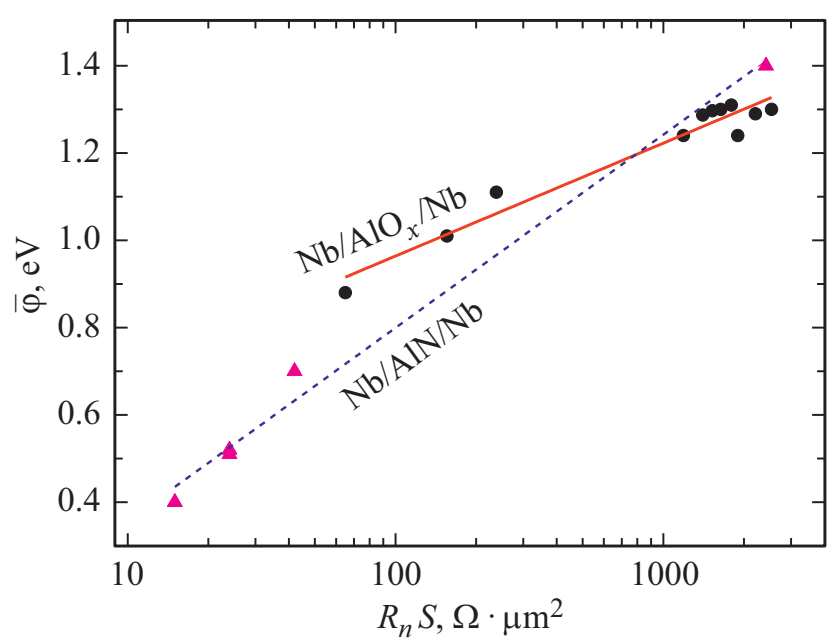

Рис. 6. Зависимость высоты туннельного барьера от значения удельного сопротивления $R_{n} S$, точки - переходы $\mathrm{Nb} / \mathrm{AlO}_{x} / \mathrm{Nb}$, треугольники - переходы $\mathrm{Nb} / \mathrm{AlN} / \mathrm{Nb}$. Сплошная и штриховая линии - линейные аппроксимации

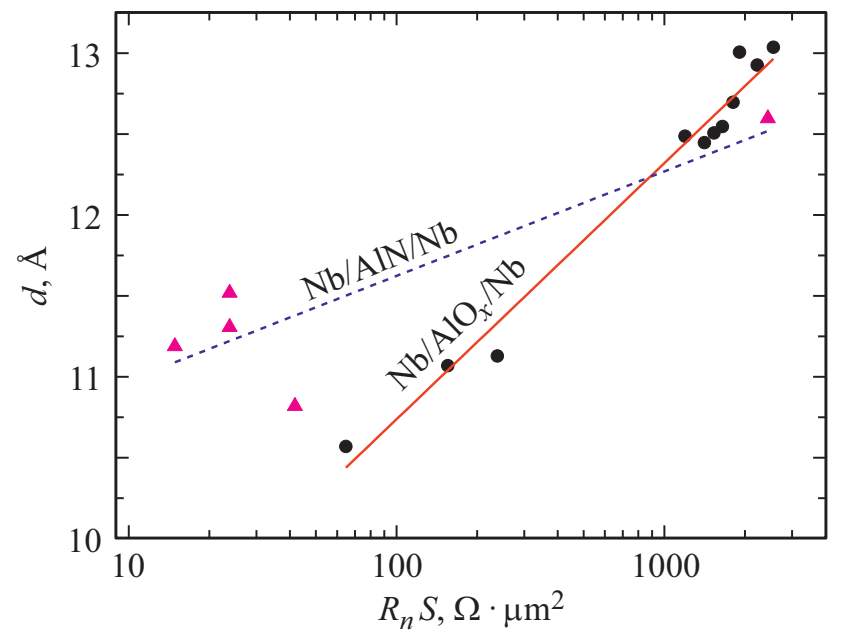

Рис. 7. Зависимость ширины туннельного барьера от значения удельного сопротивления $R_{n} S$, точки - переходы $\mathrm{Nb} / \mathrm{AlO}_{x} / \mathrm{Nb}$, треугольники - переходы $\mathrm{Nb} / \mathrm{AlN} / \mathrm{Nb}$. Сплошная и штриховая линии - линейные аппроксимации.

Для барьера AlN без поправки на эффективную массу при $R_{n} S=15 \Omega \cdot \mu \mathrm{m}^{2}$ высота барьера увеличивается до $0.5 \mathrm{eV}$, а ширина уменьшится до $7.7 \AA$. Попытки использовать расчетную модель для переходов с несимметричными электродами $(\mathrm{Nb} / \mathrm{AlN} / \mathrm{NbN})$ привели к результатам, которые существенно отличаются от параметров барьера AlN в структурах $\mathrm{Nb} / \mathrm{AlN} / \mathrm{Nb}$. Например, для $R_{n} S=1000 \Omega \cdot \mu \mathrm{m}^{2}$ высота барьера составила всего $0.7 \mathrm{eV}$, а толщина барьера $15.2 \AA$; по-видимому, для корректной оценки параметров туннельного барьера в переходах $\mathrm{Nb} / \mathrm{AlN} / \mathrm{NbN}$ необходимо использовать модели, учитывающие несимметричной характер структуры. Поэтому результаты, полученные для переходов Nb/AlN/NbN, не были представлены на рис. 6 и 7.

\section{5. Заключение}

Таким образом, в настоящей работе были измерены зависимости основных параметров барьера от величины удельного сопротивления для двух типов переходов $\mathrm{Nb} / \mathrm{AlO}_{x} / \mathrm{Nb}$ и $\mathrm{Nb} / \mathrm{AlN} / \mathrm{Nb}$. Было показано, что низкие значения высоты нитридных барьеров при высокой плотности тока (по сравнению с оксидными), позволяют реализовывать структуры с более толстыми барьерными слоями имеющими удельную проводимость не ниже $0.1 \mathrm{~S} / \mu \mathrm{m}^{2}$; это, в свою очередь, дает возможность изготовления переходов с малой утечкой при напряжениях ниже щелевого.

\section{Финансирование работы}

Исследование выполнено за счет гранта Российского научного фонда (проект № 19-19-00618). Туннельные переходы были изготовлены в ИРЭ им. В.А. Котельникова РАН в рамках государственного задания с использованием УНУ 352529. 


\section{Конфликт интересов}

Авторы заявляют, что у них нет конфликта интересов.

\section{Список литературы}

[1] J.R. Tucker, M.J. Feldman. Rev. Mod. Phys. 57, 1055 (1985).

[2] A. Karpov, J. Blondell, M. Voss, K.H. Gundlach. IEEE Transact. Appl. Supercond. 5, 3304 (1995).

[3] B.D. Jackson, G.de Lange, T. Zijlstra, M. Kroug, J.W. Kooi, J.A. Stern, T.M. Klapwijk. IEEE Transact. Microw. Theory Techn. MTT-54, 2, 547 (2006).

[4] A. Karpov, D. Miller, F. Rice, J.A. Stern, B. Bumble, H.G. LeDuc, J. Zmuidzinas. IEEE Transact. Appl. Supercond. 17, 343 (2007).

[5] A.M. Baryshev, R. Hesper, F.P. Mena, T.M. Klapwijk, T.A. Van Kempen, M.R. Hogerheijde, J. Barkhof. Astronomy Astrophys. 577, A129 (2015).

[6] A. Khudchenko, A.M. Baryshev, K.I. Rudakov, P.M. Dmitriev, R. Hesper, L. de Jong, V.P. Koshelets. IEEE Transact. Terahertz. Sci. Technol. 6, 1, 127 (2016).

[7] R.E. Miller, W.H. Mallison. Appl. Phys. Lett. 63, 10, 1423 (1993).

[8] A.W. Kleinsasser, R.E. Miller, W.H. Mallison, G.B. Arnold. Phys. Rev. Lett. 72, 11, 1738 (1994).

[9] S.K. Tolpygo, D. Yohannes, R.T. Hunt, J.A. Vivalda, D. Donnelly, D. Amparo, A.F. Kirichenko. IEEE Transact. Appl. Supercond. 17, 2, 946 (2007).

[10] M.Yu. Torgashin, V.P. Koshelets, P.N. Dmitriev, A.B. Ermakov, L.V. Filippenko, P.A. Yagoubov. IEEE Transact. Appl. Supercond. 17, 379 (2007).

[11] J. Kawamura, D. Miller, J. Chen, J. Zmuidzinas, B. Bumble, H.G. LeDuc, J.A. Stern. Appl. Phys. Lett. 76, 2119 (2000).

[12] B. Bumble, H.G. LeDuc, J.A. Stern, K.G. Megerian. IEEE Transact. Appl. Supercond. 11, 76 (2001).

[13] H.A. Huggins, M.J. Gurwitch. Appl. Phys. 57, 2103 (1985).

[14] H. Kroger, L.N. Smith, D.W. Jillie. Appl. Phys. Lett. 39, 280 (1981).

[15] P.N. Dmitriev, I.L. Lapitskaya, L.V. Filippenko, A.B. Ermakov, S.V. Shitov, G.V. Prokopenko, V.P. Koshelets. IEEE Transact. Appl. Supercond. 13, 2, 107 (2003).

[16] K.I. Rudakov, P.N. Dmitriev, A.M. Baryshev, A. Khudchenko, R. Hesperand. V.P. Koshelets. Изв. вузов „Радиофизика“ LXII, 7-8, 613 (2019).

[17] T. Imamura, S. Hasuo. IEEE Transact. Appl. Supercond. 2, 2, (1992).

[18] L.V. Filippenko, S.V. Shitov, P.N. Dmitriev, A.B. Ermakov, V.P. Koshelets, J.R. Gao. IEEE Transact. Appl. Supercond. 11, 1,816 (2001).

[19] П.Н. Дмитриев. Дис. канд. физ.-мат. наук. ИРЭ им. Котельникова РАН, Москва (2009). 96 с.

[20] Л.В. Филиппенко. Дис. канд. физ.-мат. наук. ИРЭ им. Котельникова РАН, Москва (2009). 220 с.

[21] G. deLange, D. Boersma, J. Dercksen, P. Dmitriev, A.B. Ermakov, L.V. Filippenko, H. Golstein, W.M. Ruud, L. deJongHoogeveen, A.V. Khudchenko, N.V. Kinev, O.S. Kiselev, B. van Kuik, A. deLange, J. van Rantwijk, A.S. Sobolev, M.Yu. Torgashin, EddeVries, P.A. Yagoubov, V.P. Koshelets. Supercond. Sci. Technol. 23, 4, 045016 (2010).
[22] Valery P. Koshelets, Pavel N. Dmitriev, Michael I. Faley, Lyudmila V. Filippenko, Konstantin V. Kalashnikov, Nickolay V. Kinev, Oleg S. Kiselev, Anton A. Artanov, Kirill I. Rudakov, Arno de Lange, G. de Lange, Vladimir L. Vaks, M.Y. Li, Huabing Wang. IEEE Transact. THz Sci. Technol. 5, 4, 687 (2015).

[23] N.V. Kinev, K.I. Rudakov, L.V. Filippenko, A.M. Baryshev, V.P. Koshelets. J. Appl. Phys. 125, 15, 151603 (2019).

[24] N.V. Kinev, K.I. Rudakov, L.V. Filippenko, A.M. Baryshev, V.P. Koshelets. IEEE Transact. THz Sci. Technol. 9, 6, 557 (2019).

[25] J.G. Simmons. J. Appl. Phys. 34, 6, 1793 (1963).

[26] W.F. Brinkman, R.C. Dynes, J.M. Rowell. J. Appl. Phys. 41, 5, 1915 (1970).

[27] Л.П. Булат, В.В. Конопелько, Д.А. Пшенай-Северин. Вестн. Междунар. академии холода 3, 46 (2013).

[28] М.Е. Парамонов, Л.В. Филиппенко, П.Н. Дмитриев, М.Ю. Фоминский, В.П. Кошелец. Радиотехника и электроника 64, 10, 1029 (2019).

[29] J.M. Rowell, W.L. McMillan, W.L. Feldmann. Phys. Rev. 180, 3, 658 (1969).

Редактор К.В. Емщев 